\title{
Power flow analysis of compound planetary gear trains in flight leading edge flaps
}

\author{
Fuchun Yang ${ }^{1,2, a^{*}}$, Jianxiong Feng ${ }^{1, b}$ \\ ${ }^{1}$ School of Mechanical Engineering, Shandong University, Jinan 250061, China \\ ${ }^{2}$ Key Laboratory of High-efficiency and Clean Mechanical Manufacturing at Shandong University, \\ Ministry of Education, Jinan 250061, China \\ afuchunyang@sdu.edu.cn, bjxfeng1@vt.edu
}

\begin{abstract}
Keywords: Geared rotary actuator; Planetary gear; Power flow; Hypergraph
Abstract: Geared rotary actuators in the leading edge flaps of an aircraft are usually composed of compound planetary gear sets with high reduction ratio. A new method using hypergraph in the power flow analysis of compound planetary gear sets in geared rotary actuator is proposed. The equation establishment method of kinematics and torques based on hypergraphs is investigated and the circulating power calculation approach is studied as well. A GRA on the aircraft was taken as an example to highlight the method.
\end{abstract}

\section{Introduction}

The leading edge flaps of an aircraft are hinged on the wings and can be extended with Fowler motion [1] to increase the lift coefficient of the aircraft during takeoff, to reduce landing distance during touching down and to control flight during cruise. Geared rotary actuators are usually composed of compound planetary gear sets (CPGS) with high reduction ratio. Due to the high gear ratio from the differential of compound planetary gear sets, the average efficiency is lower than that of a simple planetary gear set [2]. But the compound planetary gear sets can provide a better torque to weight ratio, which is very important for the limited space available in an aircraft. Power flow analysis is a primary procedure for efficiency calculation, especially for circulating power analysis in CPGS.

Different graphic methods were employed in the power flow analysis of planetary gear sets in the literatures [3-5]. Esmail et al [3] established a block based on a basic gear pair and a two-DOF hybrid vehicle transmission system was analyzed by this method. Apparently this method has to deal with all the gear pairs and give the relations between each element in the power flow analysis. In the analysis of a power-split planetary gear train Chen [4] applied a virtual power flow graph method and it has to deduce equations of virtual power for different gear trains. Goma Ayats et al [5] applied hypergraphs to analyze the velocity relations of one degree of freedom gear trains and this method was showed more clear and easy understanding than other methods. But it's limited to one degree of freedom systems and just used to analyze the kinematic equations.

In this paper, our study will focus on power flow analysis of GRAs with CPGSs. An approach based on hypergraphs for power flow analysis and circulating power will be proposed.

\section{Hypergraphs of planetary gear sets}

The gear trains shown in Fig.1(a) is a GRA applied in a commercial aircraft, which is composed of several CPGSs, $S_{1}, S_{2}, \ldots, S_{n}$, where $S_{i}$ is the name of $i$ th CPGS. These CPGSs have the same structure and $S_{1}$ is shown in Fig.1(b) to manifest the structure. These CPGSs are actuated by input shaft $S_{\text {in }}$ and then actuate the flaps by output rings $S_{\text {out }}$. Since the CPGSs are the same in a GRA, we need only analyze the power flow of CPGS in Fig.1(b). The CPGS in Fig.1(b) can be considered to be composed of three simple planetary gear sets (SPGTs) $a, b$ and $c$.

The fundamentals of hypergraphs used for the analysis are explained as follows. A simple planetary gear set (SPGS) as shown in Fig.2(a) typically includes three shafts (one carrier, two central gears) and three planets. A basic triangle hypergraph (BTH) is used to represent the SPGS as shown in Fig.2(b). 
In the BTH, the round node represents a shaft in the mechanism, and the line represents the mechanical connection between two shafts. The rules for the symbolization of a BTH and its nodes are given as follows: a BTH is represented by a letter, such as $x$ in Fig.2(b). The numerals 1 (sun gear) and 3 (ring gear) following a BTH's symbol represent the gear shafts, thus the combination $x 1$ represents gear 1 in the SPGS $x$; whereas numeral 2 following a BTH's symbol represents the carrier in a BTH, such as $x 2$ represents carrier 2 in the SPGS $x$. The direction of gearing is represented by an arrow and the gear ratio is put on the arrow. The letter $k$ with a BTH's name as a subscript is the ratio between gearing shaft 1 and 3 when carrier shaft 2 is fixed, that is $k_{x}=\operatorname{sign}\left(\rho_{x}\right)$, where $x$ is the BTH's name, $\rho$ is the characteristic ratio of the SPGS, $\rho_{x}=Z_{\mathrm{x} 3} / Z_{\mathrm{x} 1}, \operatorname{sign}()$ indicates the sigh function of $k$, which is determined by the meshing types between planets and central gears.

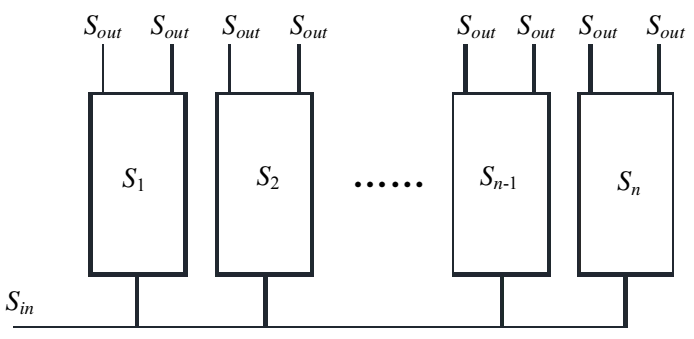

(a)

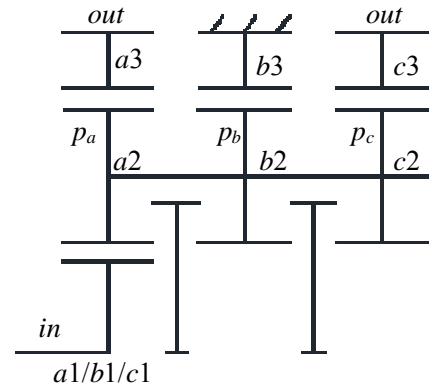

(b)

Fig.1 (a) Geared rotary actuators; (b) A compound planetary gear set $S_{1}$ of GRA (a)

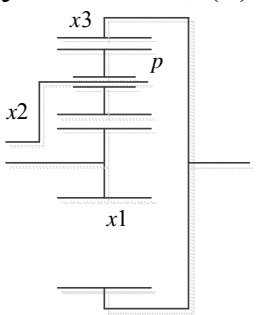

(a)

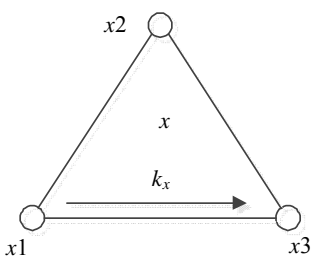

(b)

Fig.2 (a) A planetary gear set; (b) Hypergraph of SPGS

In order to analyze velocity, torque and power flow of the systems in Fig.1, hypergraph of Fig.1(b) is obtained based on the definitions above as shown in Fig.3. In Fig.3, the arrow pointing to a node represents system input, the arrow pointing out of a node represents system output and they are denoted by in and out, respectively. In a CPGS, it is typical that shafts in different SPGSs are connected together. Although the connected shaft has the same velocity in all relative SPGSs, the torque in each SPGS is different. Therefore the connected shafts are denoted by multiple overlapped circles which are defined as a group node to represent connected shafts in different BTHs, such as group nodes $a 2 \mathrm{~b} 2 \mathrm{c} 2$ in Fig.3. The node connected with ground is represented using a filled circle, such as $b 3$ in Fig.3.

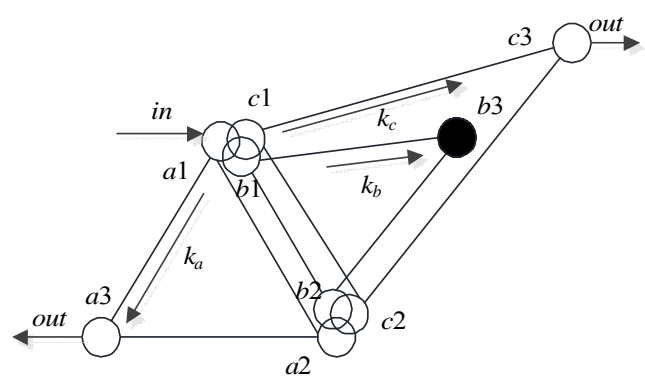

Fig.3 Hypergraph of Fig.1(b)

The velocity of each node in hypergraphs is denoted by $\omega$ with its subscript, for instance, $\omega_{a 1}$ denotes the velocity of node $a 1$. The torque for a node in each SPGS is denoted by $T$ with its subscript, for instance, $T_{b 2}$ denotes the torque in node $b 2$. The power of a node is denoted by $P$ with its subscript, for instance, $P_{b 1}$ denotes the power of node $b 1$. 


\section{Power flow analysis of planetary gear trains}

Velocity analysis. Considering kinematics of a BTH in Fig.2(b), its velocity equation can be written as following according to Willis equation [6], that is

$$
\omega_{x 1}=k_{x} \omega_{x 3}+\left(1-k_{x}\right) \omega_{x 2}
$$

Because hypergraph of a CPGS is composed of several BTHs, their velocity can be calculated according to Eq. 1 and the following procedures are generally conducted:

(1) Obtain all the velocity equations for each BTH and their boundary conditions, where boundary conditions are relationships between shafts or connections between shafts and inputs/ground;

(2) Carry out matrix operations to get the velocity of each shaft and the gear ratio of any two shafts, which is explained as follows.

Now, the velocity equations of Fig. 3 can be yielded by the foregoing rules as,

$$
\left\{\begin{array}{l}
\omega_{a 1}=k_{a} \omega_{a 3}+\left(1-k_{a}\right) \omega_{a 2} \\
\omega_{b 1}=k_{b} \omega_{b 3}+\left(1-k_{b}\right) \omega_{b 2} \\
\omega_{c 1}=k_{c} \omega_{c 3}+\left(1-k_{c}\right) \omega_{c 2} \\
\omega_{a 1}=\omega_{b 1} \\
\omega_{a 1}=\omega_{c 1} \\
\omega_{a 1}=\omega_{0} \\
\omega_{b 3}=0 \\
\omega_{a 2}=\omega_{b 2} \\
\omega_{a 2}=\omega_{c 2}
\end{array}\right.
$$

where $\omega_{0}$ is the velocity of input shaft and Eq. 2 can be rewritten in matrix form,

$$
K \omega=V
$$

where $\boldsymbol{K}$ is the coefficient matrix of characteristic ratios of SPGSs, $\boldsymbol{\omega}$ is the velocity vector and $\boldsymbol{V}$ is the constant vector.

So the velocity of each shaft can be obtained using matrix operations, that is

$$
\boldsymbol{\omega}=\boldsymbol{K}^{-1} \boldsymbol{V}
$$

Toque analysis. Ignoring the power loss in the gear train and considering force balance and power balance, the summation of torques and power on three shafts of any SPGS shown in Fig.2(b) are always zero. They are shown by the equations below, respectively,

$$
\left\{\begin{array}{l}
T_{x 1}+T_{x 2}+T_{x 3}=0 \\
T_{x 1} \omega_{x 1}+T_{x 2} \omega_{x 2}+T_{x 3} \omega_{x 3}=0
\end{array}\right.
$$

Similar to the velocity analysis, two procedures are generally to be performed as well:

(1) Obtain all the torque balance and power balance equations for each BTH and boundary conditions, where boundary conditions show whether two shafts connect to each other and input/output conditions.

(2) Carry out matrix operations to get the torques of each shaft in each BTH.

Thus, one gets all the torque equations of Fig.3 according to Eq.(5) and the above rules, it yields,

$$
\left\{\begin{array}{l}
T_{a 1}+T_{a 2}+T_{a 3}=0 \\
T_{a 1} \omega_{a 1}+T_{a 2} \omega_{a 2}+T_{a 3} \omega_{a 3}=0 \\
T_{b 1}+T_{b 2}+T_{b 3}=0 \\
T_{b 1} \omega_{b 1}+T_{b 2} \omega_{b 2}+T_{b 3} \omega_{b 3}=0 \\
T_{c 1}+T_{c 2}+T_{c 3}=0 \\
T_{c 1} \omega_{c 1}+T_{c 2} \omega_{c 2}+T_{c 3} \omega_{c 3}=0 \\
T_{a 2}+T_{b 2}+T_{c 2}=0 \\
T_{a 3}=\gamma T_{c 3} \\
T_{a 3}+T_{c 3}=T_{0}
\end{array}\right.
$$


where $\gamma$ is the load distribution ratio of two outputs in Fig. 3 and $T_{0}$ is the total output torque of the system. Then Eq. 6 can be rewritten in matrix form,

$$
M T=D
$$

where $\boldsymbol{M}$ is the matrix of velocities obtained in Eq. $4, \boldsymbol{T}$ is the torque vector and $\boldsymbol{D}$ is the constant vector. Then the torques on all shafts can be obtained from the equation below,

$$
\boldsymbol{T}=\boldsymbol{M}^{-1} \boldsymbol{D}
$$

Power flow. From the above analysis, powers of each node for all BTHs can be obtained, that is

$$
\boldsymbol{P}=\boldsymbol{T} \omega
$$

where $\boldsymbol{P}$ is the power vector.

The power computed by Eq. 9 is signed values and its directions are given by the following rules:

If $P_{i j}>0$, power flows into the BTH by node $i j$,

If $P_{i j}<0$, power flows out of the BTH by node $i j$,

If $P_{i j}=0$, power flows through node $i j$.

Circulating power. Circulating power in a planetary gear set will cause extra power losses and increase the forces on components. Therefore, it is very important to identify the circulating power in planetary gear sets. The approach to calculate the circulating power can be done as following:

1) Search from an input node in a PGT as the original node, the input should not be the system input. If the input node connects with the system input and its power is bigger than the system input, then the input power for this PGT should be the power of this node minus the power of the system input;

2) Following the power flow, find out the node whose power is equal or bigger than the power of the original node. If the searching process returns to the original node, the searched nodes comprise a circulating route and the circulating power equals to the original node power.

\section{Example and Conclusions}

Example. In this section, an example will be applied to manifest the method proposed above. Fig. 1 is a typical geared rotary actuator composed of compound planetary gear sets in flight control system. The power flow analysis of Fig. 1 will be investigated and the parameter influence, such as character ration, load coefficient, etc., on circulating power and system input power will be studied as well. The basic parameters for CPGS in Fig.1(b) are shown in Table 1.

Table 1 Basic parameter of the system

\begin{tabular}{llllclll}
\hline & $a 1 / b 1 / c 1$ & $a 3$ & $b 3$ & $c 3$ & $P_{a}$ & $P_{b}$ & $P_{c}$ \\
\hline Teeth & 21 & 180 & 165 & 180 & 80 & 65 & 80 \\
number & & & & & & & \\
$m[\mathrm{~mm}]$ & & & & 1 & & & \\
$\alpha\left[^{\circ}\right]$ & & & & 20 & & & \\
Gear ratio & & & & 83.229 & & & \\
$\rho_{a}, \rho_{c}$ & & & & 8.57 & & & \\
$\rho_{b}$ & & & & 9.67 & & & \\
\hline
\end{tabular}

In order to analyze the power flow of the system, the system input velocity is set to 500rpm, the total load on output rings in Fig.1(b) is set to $2000 \mathrm{Nm}$ and $\gamma=1$. Based on velocity, torque and power equations Eq. 4, Eq. 8, Eq. 9, the normalized powers to input power of each component in Fig.3 are obtained as shown in Fig.4. From Fig. 4 one can see that the powers on sun gears of three simple planetary gear sets are different. However, their summation equals to system input power because they are combined as one component physically. The output powers of ring $a 3$ and ring $c 3$ equals to half of the system input power because the load distribution ratio $\gamma=1$. The powers on three carriers are much higher than system input power and the power on the carrier of simple planetary gear set $b$ is different from that of the other two simple planetary gear sets. Although the three carriers connect together 
physically, there are inner interaction among them, which may cause heavy load on carrier bearings and reduce the strength of planet gear pin and the life of bearings.

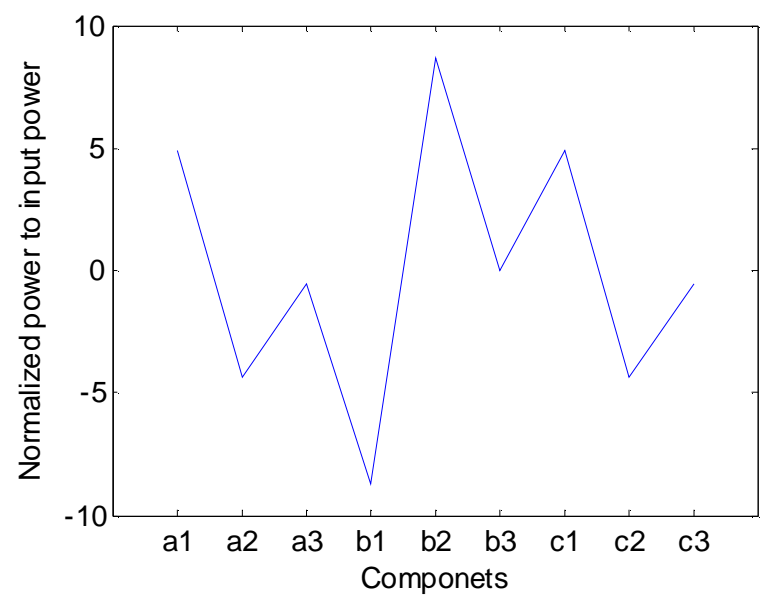

Fig.4 Normalized power of each component in Fig.3

Fig. 5 is the power flow of the system based on the results in Fig. 4 and powers flow along the arrow lines. The input power flows into $a 1 / b 1 / c 1$ component, then the power in $a 1$ is split into two paths, that is $a 3$ and $a 2$, as such, the power in $c 1$ is split into two paths $c 2$ and $c 3$. The powers of $a 3$ and $c 3$ are system output power, while the powers of $a 2$ and $c 2$ are combined to $b 2$ and then flow back to $b 1$. This means there are two circulating powers in this system, one is $a 1-a 2-b 2-b 1-a 1$ and the other is $c 1-c 2-b 2-b 1-c 1$. The circulating power is about ten times of system input power. This high circulating power will cause additional power losses and reduce the strength and life of some components. Therefore, designer should reduce the circulating power in this system. In addition, this kind of circulating power cannot be found by traditional analysis method using formulas and it might cause the planet gear tilting in the GRA which could induce the heavy wear between the planet gear and the carrier.

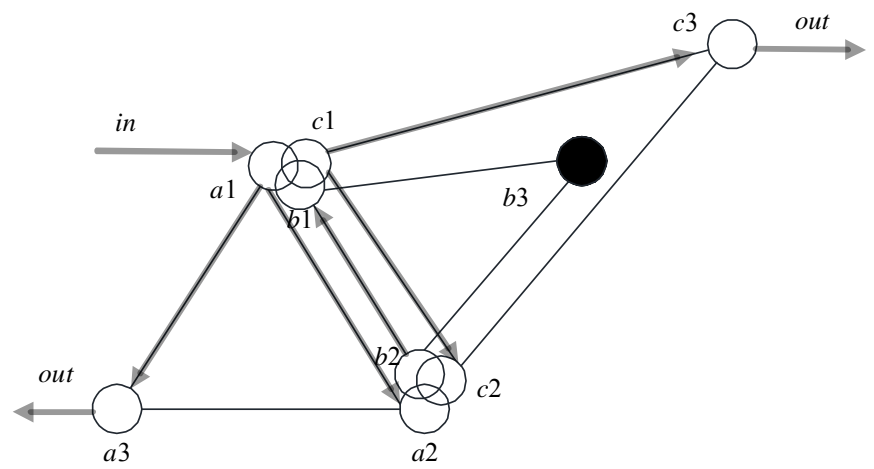

Fig.5 Power flow of Fig.3

Characteristic ratios are important parameters in the design of compound planetary gear sets. Here, the influence of characteristic ratios on circulating power will be investigated and a reasonable range of characteristic ratios for the system will be determined. In order to keep the system gear ratio constant around 83.229, the characteristic ratio of planetary gear set $b$ will change with the increase of that of planetary gear set $a$. It is obvious that the normalized circulating power decreases with the increase of characteristic ratio $\rho_{a}$ as shown in Fig.6(a). It will reduce about $20 \%$ circulating power if the characteristic ratio $\rho_{a}$ equals to 9 instead the given value 8.57 in Table 1. From Fig.6(b) it can be observed that the characteristic ratio $\rho_{b}$ has almost linear relationship with $\rho_{a}$ to keep the system gear ratio constant. Hence, increasing the characteristic ratio of the system is an effective way to reduce system circulating power. 


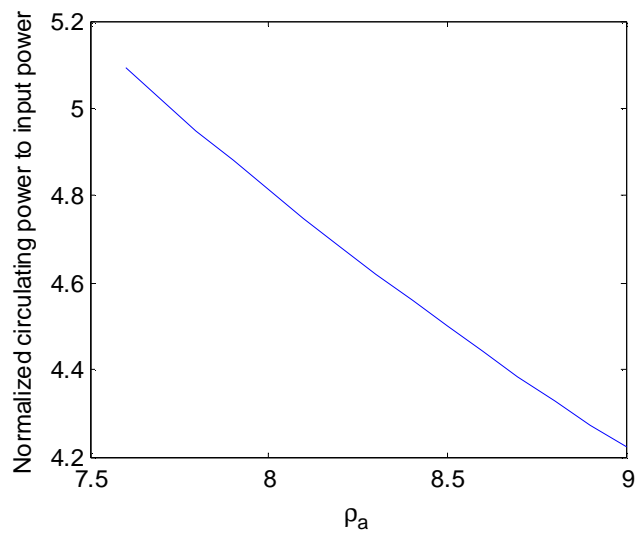

(a)

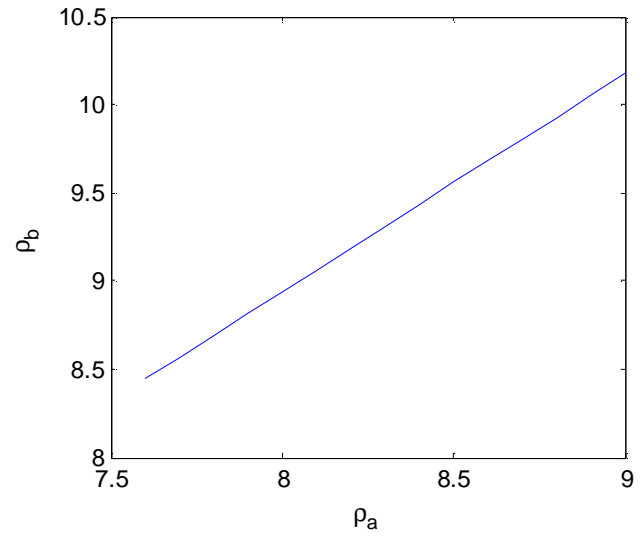

(b)

Fig.6 (a) Relation between circulating power and characteristic ratio $k_{a}$; (b) $k_{b}$ vs. $k_{a}$ by keeping $R$ around 83.229

Conclusions. In this paper, a new method using hypergraph in the power flow analysis of compound planetary gear sets in geared rotary actuator is proposed. The equation establishment method of kinematics and torques based on hypergraph was investigated and the circulating power calculation approach was also studied. A GRA on the aircraft was taken as an example to highlight the method. The influence of parameters on power flow and circulating power was also investigated.

\section{Acknowledgements}

This paper was supported by National Natural Science Foundation of China (Grant No.51305233), Research Fund for the Doctoral Program of Higher Education of China (Grant No.20120131120056) and Independent Innovation Foundation of Shandong University (Grant No.2012TS048).

\section{References}

[1] P.K.C. Rudolph. High-lift system on commercial subsonic airliners. NASA Contractor Report, 1996, 4746.

[2] A., Wang, S., Gitnes and L., El-Bayoumy. The instantaneous efficiency of epicyclic gears in flight control systems. Journal of mechanical design, 2011, 133: 051008.

[3] Essam L. Esmail, Shaker S. Hassan. An Approach to Power-Flow and Static Force Analysis in Multi-Input Multi-Output Epicyclic-Type Transmission Trains. Journal of mechanical design, 2010, 132(011009): 1-10.

[4] Chen. Power flow and efficiency analysis of epicyclic gear transmission with split power. Mechanism and machine theory, 2013, 59: 96-106.

[5] J.R. Goma Ayats, U. Diego-Ayala, J. Minguella Canela, F. Fenollosa, J. Vivancos. Hypergraphs for the analysis of complex mechanisms comprising planetary gear trains and other variable or fixed transmissions. Mechanism and machine theory, 2012, 51:217-229.

[6] Robert Willis. Principles of mechanism. Parker, London, 1841. 